\title{
Use of physical restraints in nursing homes: a multicentre cross-sectional study
}

Hedi Hofmann ${ }^{1,2^{*}}$, Ewald Schorro ${ }^{3,2 \dagger}$, Burkhard Haastert ${ }^{4}$ and Gabriele Meyer ${ }^{5,2}$

\begin{abstract}
Background: Although many countries have implemented strict legal rules, the prevalence of physical restraints in nursing homes seems to remain high. In Switzerland, data related to the frequency of physical restraints are scarce and little is known about associations with resident and nursing home characteristics. The aim of this study was to investigate the prevalence and types of physical restraints in nursing homes in two Swiss cantons and to explore whether resident-related and organisational factors are associated with the use of physical restraints.
\end{abstract}

Methods: We conducted a multicentre cross-sectional study. Twenty nursing homes with 1362 residents from two culturally different cantons were included. Data on physical restraints and residents' characteristics were extracted from residents' records (11/2013 to 2/2014). Organisational data were collected by questionnaires addressing nursing home directors or nursing managers. Sample size calculation and outcome analysis took cluster-adjustment into account. Descriptive statistics and multiple logistic regression analysis with nursing homes as random effect were used for investigation.

Results: The prevalence of residents with at least one physical restraint was $26.8 \%$ (95\% confidence interval [Cl] 19.8-33.8). Centre prevalence ranged from 2.6 to $61.2 \%$. Bilateral bedrails were most frequently used (20.3\%, $95 \%$ Cl 13.5-27.1). Length of residence, degrees of care dependency and mobility limitation were significantly positively associated with the use of physical restraint, but none of the organisational characteristics was significantly associated.

Conclusion: Approximately a quarter of the nursing home residents included in our study experienced physical restraints. Since variation between nursing homes was pronounced, it seems to be worthwhile to explore nursing homes with particularly low and high use of physical restraints in future research, especially by using qualitative methods. There is a need for effective interventions aiming at restraint-free nursing care. Development of interventional approaches should consider specific residents' characteristics associated with restraint use.

Keywords: Nursing, Physical restraint, Nursing homes, Epidemiology, Prevalence

\section{Background}

The use of physical restraints in nursing homes has been the subject of numerous empirical studies and discussion papers during recent years. Even though there are strict legal regulations in many countries, the prevalence of physical restraints in nursing homes seems still to be high $[1,2]$. Due to methodological reasons and different

\footnotetext{
* Correspondence: hedi.hofmann@fhsg.ch

${ }^{\dagger}$ Equal contributors

'Department of Health, University of Applied Sciences, Rosenbergstrasse 59,

P.O. BoxCH-9001 St.Gallen, Switzerland

${ }^{2}$ Department of Nursing Science, Witten/Herdecke University, Witten,

Germany

Full list of author information is available at the end of the article
}

definitions of physical restraints [3], a wide variation in the reported prevalence could be found. However, crossnational data by Feng et al. [4], using standardised methods for data collection in five countries, suggested a range of prevalence from 6 to $31 \%$. In Switzerland, data were collected in 94 nursing homes and revealed a prevalence of $6 \%$. Bedrails were excluded and all five participating cantons (i.e. member states of the Swiss Confederation) belonged to the German-speaking part of the country [4]. In another Swiss study data were collected on various quality indicators in nursing homes and a bedrail prevalence of $18.5 \%$ was found [5]. Studies conducted in Germany $[2,6]$ reported prevalence figures

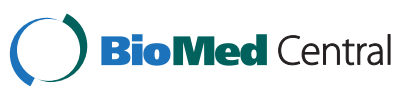

(c) 2015 Hofmann et al. Open Access This article is distributed under the terms of the Creative Commons Attribution 4.0 International License (http://creativecommons.org/licenses/by/4.0/), which permits unrestricted use, distribution, and reproduction in any medium, provided you give appropriate credit to the original author(s) and the source, provide a link to the Creative Commons license, and indicate if changes were made. The Creative Commons Public Domain Dedication waiver (http://creativecommons.org/publicdomain/zero/1.0/) applies to the data made available in this article, unless otherwise stated. 
of approximately $30 \%$. Variation of physical restraint prevalence between nursing homes seems to be pronounced, as indicated by Meyer et al. [6], reporting a range between 4 and $59 \%$ residents with at least one physical restraint. According to a widely accepted definition, we understand physical restraint as 'any device, material or equipment attached to or near a person's body and which cannot be controlled or easily removed by the person and which deliberately prevents or is deliberately intended to prevent a person's free body movement to a position of choice and/or a person's normal access to their body' [7].

From an ethical perspective, the use of physical restraints is highly questionable since the devices constrict residents' dignity and autonomy and thereby harm personal integrity [8]. Decisions about the use of physical restraints in the care of older persons are complex and influenced by different factors like attitudes of health care staff, characteristics of residents as well as nursing home and environmental characteristics [9]. Even when nurses have negative feelings towards physical restraints, they identify a need for their use in specific situations and for various reasons like fall prevention or control of challenging behaviour [10]. The risk of using physical restraints is increased in residents with a low level of activities of daily living (ADL). Low cognitive function, repeated verbal and physical agitation as well as severe mobility impairments, previous accidental falls and related fractures are further factors associated with the use of physical restraints $[3,11]$. In nursing homes with dementiaspecific care units, special quality improvement strategies and public ownership, physical restraints seem to be used less often $[9,12,13]$. Staffing level seems not to be associated with the use of physical restraints $[9,11]$.

Physical restraints are associated with various negative outcomes like reduced residents' quality of life, worse physiological and psychological condition [1], decrease of physical activity level, reduction of cognitive functioning, worsening of dementia-related behavioural symptoms [14] as well as increase of accidental falls, pressure ulcers and incontinence [3]. The majority of serious direct injuries from bedrails are caused by incorrect application or use of non-appropriate devices. Evidence on health benefits through the application of physical restraints, such as prevention of injuries, are lacking [15].

Various interventions to reduce physical restraints in nursing homes have been developed [16]. Recently published studies indicate the effectiveness of multifactorial preventive approaches. Physical restraints were reduced [17] and the use of belts decreased significantly [18, 19], also in newly admitted residents [20].

In Switzerland, according to a new law valid since January 2013, the use of physical restraints is allowed under exceptional circumstances only for residents who are at risk of harming themselves or others [21]. Data concerning the frequency of physical restraint use in Swiss nursing homes are scarce and little is known especially about the application of bedrails. Country-based prevalence data are required for a better understanding of the practice of physical restraint use and for decisionmaking about the need for interventions to reduce physical restraints. In the present study, we aimed to determine how often all types of physical restraints are applied among nursing home residents in two Swiss cantons, St.Gallen and Fribourg, and whether specific characteristics of residents and nursing homes are associated with a higher likelihood of restraint use. According to the federal structure of Switzerland, a dominant part of the health care system is regulated at the cantonal level, particularly in the case of nursing homes. Nursing practice in the cantons is also influenced by the respective language culture. There is evidence that differences in regulation and common practice may result in differences of physical restraint use [22]. St.Gallen belongs to German-speaking Switzerland and Fribourg to French-speaking Switzerland (Romandy) closely associated with French culture and the Roman as well as Latin tradition.

\section{Methods}

\section{Design}

We conducted a multicentre cross-sectional study.

\section{Sample/Participants}

One hundred and eight nursing homes with at least 30 beds were eligible for inclusion. In a first step we built strata considering the characteristics "canton", "size of nursing home", and "rural or urban environment". From these strata, nursing homes were randomly selected and consecutively invited until 20 institutions with a total of 1362 residents agreed to participate. Twelve institutions denied participation due to lack of time, nursing shortage, refurbishment and lack of interest. In total, we sent 32 invitation letters and gave 21 personal presentations about the study. Recruitment took place from July 2013 to January 2014. In each nursing home, a responsible nurse for the coordination of the study was nominated.

Based on Feng et al. [4] and Meyer et al. [6] the mean prevalence of physical restraints (including bedrails) was assumed to be $25 \%$. Experts and nursing home directors $(n=5)$ in Switzerland judged this figure as realistic. Without cluster adjustment a sample size of 214 would have been sufficient to reach a $95 \%$ confidence interval of 25 $\% \pm 6 \%=19-31 \%$. For proper cluster adjustment, we applied an intracluster correlation coefficient (ICCC) of 0.08 , according to Meyer et al. [6]. Assuming a mean cluster size (=number of participants per home) of 68 , a design factor of DF $=1+(\mathrm{m}-1) \times$ ICCC $[\mathrm{m}=$ mean cluster size $]=$ $1+(68-1) \times 0.08=6.36$ is concluded [23]. Distributing 
$6.36 * 214=1361$ residents over homes with mean cluster sizes of 68 overall 20 homes are needed.

\section{Data collection}

Data collection was performed between November 2013 and February 2014. Data were obtained by trained external investigators (HH, ES, two study assistants) using the web-based software SecuTrial ${ }^{\circ}$.

Data collection on physical restraints covered items taken from the Maastricht Attitude Questionnaire (MAQ) $[24,25]$ as bilateral bedrails, unilateral bedrail at one side of the bed with the other positioned at the wall, belt in bed, sleep suit, tight sheet (a sheet over belly and upper legs that is tightened firmly under the mattress at both sides of the bed), wrist restraint, ankle restraint, chair preventing rising (deep or overturned chair), chair with a locked tray table, wheelchair with a locked tray table and belt in chair. The nominated nurses gathered data from the residents' BESA or RAI records $[26,27]$ and nursing reports. In Switzerland, BESA (in German: Bedarfsklärungs- und AbrechnungsSystem, in English: Needs Assessment and Payoff System) [26] and RAI (Resident Assessment Instrument) [27] are two established standard tools to assess residents' care needs and resources and to document nursing services. According to the Swiss federal law on the reorganization of care funding [28], twelve different degrees of care dependency can be distinguished. Degree one is related to the lowest care dependency and degree twelve to the highest. BESA and RAI data have to be updated every six month since nurses in Switzerland are obliged to regularly assess residents' conditions and needs. Data considering age, gender, degree of care dependency, length of stay in the nursing home, mobility restrictions, accidental falls, and fall-related fractures were collected.

Data concerning nursing home characteristics covered ownership, number of beds, presence of dementia-specific care units, staffing ratio, skills-/grade-mix of the nursing team, in-house or external physicians, access control to the nursing home, availability of guidelines and documentation standards aimed to control the use of physical restraints. These data were collected by questionnaires addressing nursing home directors or nursing managers of the 20 participating institutions.

\section{Ethical considerations}

The ethics committee of the Canton of St.Gallen and the ethics committee for research of the Canton of Fribourg approved the study protocol. The approval covered all participating centers. The external investigators had neither direct access to the residents' records nor personal contact with the residents. Residents were not identified by name during data collection and analysis. Thus, data protection was fully guaranteed. According to the ethics committees, no additional informed consent was required from the nursing home directors completing the questionnaires.

\section{Data analysis}

Baseline characteristics of nursing homes and residents were expressed as means \pm standard deviations (SD), numbers, and percentages. The primary outcome of the study was prevalence of restraints (resident with at least one physical restraint). All parameters describing the use of physical restraints as well as resident-related and organisation-related factors were considered as outcomes. These outcome variables are correlated within the clusters, such that simple variance estimators are biased (too low). Variances and confidence intervals for prevalence were adjusted for cluster correlation using methods described by Donner and Klar [23]. In case of unequal cluster sizes (cluster sizes vary between 36 and 164) the use of minimum variance weights was recommended by Kerry and Bland [29] because of a smaller increase of the variance due to the design effect from clustering. For the outcome variables, the cluster-correlation was estimated by the corresponding intra-cluster correlation coefficient (ICCC) and the design factor DF which depends on ICCC, cluster structure and weights. Finally, cluster-adjusted approximate two-sided $95 \%$ confidence intervals (CI) for prevalence were calculated.

Associations between resident-related and organisationrelated factors and physical restraint use were investigated by generalized linear mixed (logistic) models [30]. The binary variable "restraint yes/no" was the dependent variable and baseline variables were the independent variables as fixed effects. Cluster-adjustment was achieved by including clusters as random effects. In these models clusteradjusted odds ratios (AORs) were estimated.

A set of baseline variables (Table 4) on nursing homes and residents' characteristics were evaluated in clusteradjusted univariate models (i.e. for each variable one separate model). Additionally, one big multiple model was fitted, including all variables. All significant variables from the univariate models and the big multiple model were selected for a final model. Significant variables from univariate models not being significant in the big multiple model were excluded. Furthermore, data concerning sex, age and agitation were selected for epidemiological or clinical reasons.

The level of significance was $5 \%$. All tests and confidence intervals were two-sided. Statistical analysis was performed using SPSS 21 and SAS 9.4.

\section{Rigour}

To ensure completeness and correctness, a random sample of $20 \%$ of the data were independently checked by two investigators. The absolute agreement of this check was $96.3 \%$. The entire data set was checked on plausibility 
in a systematic procedure by the biometrician of the study (BH).

\section{Results}

Baseline characteristics of nursing homes and residents are displayed in Tables 1 and 2. Two third of the nursing homes were located in a rural area, $70 \%$ had a nonprofit ownership and the average number of beds per home was $n=70$. Residents' age ranged from 42 to 105 years, with a mean age of 85.1 (SD 8.4) and the median age of $86.0 ; 71.7 \%$ of residents were female. Approximately $70 \%$ of the residents were allocated to the degrees of

Table 1 Baseline characteristics of nursing homes $(n=20)$

\begin{tabular}{ll}
\hline Characteristics & \\
\hline Region & $12(60)$ \\
St.Gallen & $8(40)$ \\
Fribourg & \\
Location & $7(35)$ \\
$\quad$ Urban & $13(65)$ \\
$\quad$ Rural & \\
Ownership & $14(70)$ \\
$\quad$ Non-profit & $5(25)$ \\
$\quad$ Private & $1(5)$ \\
Other & $7(35)$ \\
Nursing homes with $\geq 1$ dementia \\
care unit
\end{tabular}

Values are numbers (percentage) or mean \pm standard deviation (range). ${ }^{\mathrm{a}} F T E$ full time equivalent; ${ }^{b}$ percentage from 12 homes with in-house standard
Table 2 Baseline characteristics of nursing homes residents ${ }^{a}$ $(n=1362)$

\begin{tabular}{|c|c|}
\hline Characteristics & \\
\hline \multicolumn{2}{|l|}{ Region } \\
\hline St.Gallen & $844(62)$ \\
\hline Fribourg & $518(38)$ \\
\hline Women & $977(71.7)$ \\
\hline Age, years, mean $\pm S D$ (range) & $85.1 \pm 8.4(42-105)$ \\
\hline $\begin{array}{l}\text { Length of residence, years, range } \\
\text { (lower quartile, median, upper quartile) }\end{array}$ & $0-60.4(1.0,2.3,4.4)$ \\
\hline \multicolumn{2}{|l|}{ Degree of care dependency } \\
\hline $1-3$ & $351(25.8)$ \\
\hline $4-6$ & $408(30.0)$ \\
\hline $7-9$ & $518(38.0)$ \\
\hline $10-12$ & $85(6.2)$ \\
\hline Mobility limitation ${ }^{b}$ & $744(56.2)$ \\
\hline Verbal agitation $^{c}$ & $303(22.3)$ \\
\hline Physical agitation $^{d}$ & $172(12.7)$ \\
\hline Fall during preceding 30 days $^{e}$ & $202(14.8)$ \\
\hline Fall during preceding $>30$ to 180 days $^{\mathrm{e}}$ & $349(25.6)$ \\
\hline Hip fracture during preceding 180 days $^{f}$ & $16(1.2)$ \\
\hline Other fracture during preceding 180 days $s^{f}$ & $25(1.8)$ \\
\hline
\end{tabular}

Values are numbers (percentage) unless stated otherwise. ${ }^{a}$ Not cluster adjusted; ${ }^{b} n=38$ missings; ${ }^{c} n=1$ missing; ${ }^{\mathrm{d}} n=2$ missings; ${ }^{\mathrm{e}} n=89$ residents fulfilled both fall criteria and are counted twice; ${ }^{f} n=1$ resident fulfilled both fracture criteria and was counted twice

care dependency four to nine, indicating a medium to high level of care dependency.

\section{Prevalence and types of physical restraints}

The cluster-adjusted prevalence of residents with at least one physical restraint was $26.8 \%$ [95\% confidence interval (CI) 19.8-33.8], only slightly higher as expected. Centre prevalence ranged from 2.6 to $61.2 \%$. Bilateral bedrails and unilateral bedrails at one side of the bed with the other positioned at the wall were the most frequently used physical restraints [20.3 \% (95 \% CI 13.527.1 ) and $5.7 \%$ (95 \% CI 2.7-8.8)], followed by wheelchair with a locked tray table $[1.8 \%$ (95\% CI 0.6-3.1)], belt in chair [1.1 \% (95\% CI 0.4-1.9)], and sleep suits [1.1 \% (95 \% CI 0.4-1.8)]. Further methods of physical restraints were scarcely or not reported at all (Table 3 ). Cluster adjusted prevalences in St.Gallen and Fribourg were $27.6 \%$ (95 \% CI 16.8-38.4) and $25.9 \%$ (95\% CI $18.6-33.3 \%$ ), $p=0.993$ (cf. univariate model in Table 4). The estimated intra-cluster correlation coefficients (ICCC) are presented in Table 3. The ICCC for the overall prevalence of 0.12 was higher than assumed in the sample size estimation, such that cluster adjustment results in larger variance estimations and CIs. 
Table 3 Frequency of physical restraints

\begin{tabular}{|c|c|c|c|}
\hline & $\begin{array}{l}\text { Cluster-adjusted } \\
\text { prevalence, \% (95\% Cl) }\end{array}$ & $\mathrm{ICCC}^{\mathrm{a}}$ & $\mathrm{DF}^{\mathrm{b}}$ \\
\hline $\begin{array}{l}\text { Residents with at least } \\
\text { one physical restraint }\end{array}$ & $26.8(19.8-33.8)$ & 0.1168 & 8.958 \\
\hline Bilateral bedrails & $20.3(13.5-27.1)$ & 0.1319 & 9.973 \\
\hline $\begin{array}{l}\text { Unilateral bedrail at one side } \\
\text { of the bed with the other } \\
\text { positioned at the wall }\end{array}$ & $5.7(2.7-8.8)$ & 0.0847 & 6.805 \\
\hline Sleep suits & $1.1(0.4-1.8)$ & 0.0092 & 1.684 \\
\hline Belt in bed & $0.1(0.0-0.3)$ & 0.0066 & 1.496 \\
\hline Belt in chair & $1.1(0.4-1.9)$ & 0.0133 & 1.969 \\
\hline Chair preventing rising & $0.5(0.0-1.2)$ & 0.0190 & 2.366 \\
\hline Chair with a locked tray table & $0.3(0.0-0.6)$ & 0.0005 & 1.039 \\
\hline $\begin{array}{l}\text { Wheelchair with a locked } \\
\text { tray table }\end{array}$ & $1.8(0.6-3.1)$ & 0.0315 & 3.218 \\
\hline
\end{tabular}

${ }^{\mathrm{a}}$ ICCC Intracluster correlation coefficient, ${ }^{\mathrm{b}} D F$ Design factor. Due to low prevalence of physical restraints (all outcomes except all physical restraints and bedrails on both sides) the asymptotical estimation of cluster-adjusted $95 \%$ $\mathrm{Cl}$ should be interpreted with caution

\section{Associations with the use of physical restraints}

Table 4 displays the results of the cluster-adjusted univariate and multiple logistic regression analyses. Most significant univariate associations remain significant in the multiple model adjusted for the other variables with some changes of the estimated odds ratio. Only the positive association of the number of residents per caregiver [full time equivalent (FTE) $(\leq 1.7$ vs. $>1.7)$ ] in the univariate model (AOR 2.39, 95 \% CI 1.14-5.05) was explained by the other variables in the big model containing all variables. In the final multiple model with selected variables, length of residence for more than 4.5 years (AOR $1.91,95 \%$ CI 1.22-3.02), degree of care dependency of 4-6 (AOR 9.93, 95 \% CI 3.63-27.13), degree of care dependency of 7-9 (AOR 50.06, $95 \%$ CI 18.54-135.15), degree of care dependency of 10-12 (AOR 294.25, $95 \%$ CI 94.37-917.45) and mobility limitations (AOR 3.46, $95 \%$ CI 2.35-5.10) are positively and significantly associated with the use of physical restraints. The variable "cognition" was not documented in a proper way in the BESA/ RAI records and only few residents had either a diagnosis of dementia or a Mini-Mental State Examination. Therefore, data on cognitive status were incomplete and not suitable for inclusion into the analysis.

None of the organisational characteristics was significantly associated with a higher probability of physical restraint use.

\section{Discussion}

Our cross-sectional study in two Swiss cantons indicates that nearly 27 of 100 residents were subject to at least one physical restraint. The pronounced variation of prevalence between nursing homes (2.6\% to $61.2 \%)$ is in accordance with studies from Germany [6]. A recent European study with nursing home residents suffering from dementia in eight countries revealed a prevalence of physical restraints of $31.4 \%$ with country variation ranging from 6.1 to $83.2 \%$ [31]. Thus, a relation between nursing practice regarding physical restraints and cultural background is likely. Our study revealed no difference in the prevalence between the German-speaking and the French-speaking part of Switzerland. The enactment of the new Swiss-wide law in 2013 might have minimized any differences in the use of physical restraints. However, this is only speculation, since there is no previous prevalence study comparing two culturally different regions of Switzerland.

Bedrails were the most commonly used type of physical restraint, as reported by a recent study on nursing home quality in Switzerland [5]. The former study revealed a lower prevalence figure. Different data collection methods might have led to an underestimated prevalence. As described by earlier studies, nurses judge bilateral bedrails as a moderate restrictive measure and they do not hesitate to use them [24]. Therefore, one might assume that the application of physical restraints in some nursing homes is still routine care and used in order to prevent falls and ensure safety. However, bedrails do not always guarantee residents' safety. On the contrary, as shown in other studies, they can also cause injuries and even death of residents by entrapment [32]. Limiting a resident's movement by using chairs with a locked tray table was a rare type of physical restraint in our study. Belts in chairs or sleep suits were also seldom applied.

We found significant resident-related associations with the application of physical restraints, which were predominately bedrails. Increased degree of care dependency as well as mobility limitations revealed pronounced positive associations with restraint use. Earlier studies confirm these results $[3,5,6]$. Considering residents' baseline characteristics, with $75 \%$ of the residents displaying a higher degree of care dependency and more than half of the persons with mobility limitations, the probability of physical restraint is therefore notably elevated. An increased length of residence in the institution was also positively associated with the use of physical restraint. These results are confirmed by the cross-national study by Feng et al. [4] who described an association between an extended period of institutionalisation and the risk of restraint use in Switzerland. However, data from other participating countries (Canada, Hong Kong, Finland and USA) did not show any relation between longer resident stay and increased risk of physical restraint [4]. Our results do not indicate positive associations with gender or older age as described by former studies [11,33]. Verbal and physical agitation was not associated with restraint use. As the baseline data show, these characteristics were 
Table 4 Cluster-adjusted logistic regression models (cluster = random effect)

\begin{tabular}{|c|c|c|c|c|}
\hline \multirow[b]{2}{*}{ Variable } & \multicolumn{2}{|l|}{ Univariate models } & \multicolumn{2}{|l|}{ Multiple model } \\
\hline & Odds ratio $(95 \% \mathrm{Cl})$ & $p$-value & Odds ratio $(95 \% \mathrm{Cl})$ & $p$-value \\
\hline \multicolumn{5}{|l|}{ Nursing home characteristics } \\
\hline Region, Fribourg vs. St.Gallen & $1.00(0.42-2.42)$ & 0.993 & & \\
\hline Location, rural vs. urban & $0.92(0.37-2.27)$ & 0.848 & & \\
\hline \multicolumn{5}{|l|}{ Ownership } \\
\hline Private vs. non-profit & $2.39(0.98-5.83)$ & 0.055 & & \\
\hline Other vs. non-profit & $3.36(0.59-18.97)$ & 0.155 & & \\
\hline Dementia care unit, yes vs. no & $1.13(0.46-2.78)$ & 0.773 & & \\
\hline Residents per nursing home, $\leq 70$ vs. $>70$ & $0.90(0.37-2.20)$ & 0.803 & & \\
\hline Residents per caregiver FTE ${ }^{a}, \leq 1.7$ vs. $>1.7$ & $2.39(1.13-5.05)$ & 0.025 & & \\
\hline Residents per night nurse, $\leq 30$ vs. $>30$ & $1.76(0.78-3.96)$ & 0.159 & & \\
\hline Proportion of trained nursing staff, $\leq 40$ vs. $>40$ & $0.77(0.32-1.83)$ & 0.533 & & \\
\hline \multicolumn{5}{|l|}{ Medical care } \\
\hline General practitioner vs. in-house physician & $0.39(0.09-1.67)$ & 0.187 & & \\
\hline Others vs. in-house physician & $0.52(0.12-2.24)$ & 0.354 & & \\
\hline Physical restraint standard with definition, yes vs. no & $1.08(0.68-1.72)$ & 0.735 & & \\
\hline \multicolumn{5}{|l|}{ Resident characteristics } \\
\hline Gender, men vs. women & $1.00(0.75-1.31)$ & 0.975 & $1.27(0.89-1.82)$ & 0.189 \\
\hline \multicolumn{5}{|l|}{ Age } \\
\hline $75-84$ vs. $<75$ years & $0.76(0.49-1.17)$ & 0.213 & $0.87(0.50-1.50)$ & 0.606 \\
\hline $85-94$ vs. $<75$ years & $0.92(0.62-1.38)$ & 0.701 & $1.27(0.75-2.14)$ & 0.369 \\
\hline$\geq 95$ vs. $<75$ years & $1.14(0.66-2.00)$ & 0.634 & $1.49(0.72-3.08)$ & 0.283 \\
\hline \multicolumn{5}{|l|}{ Length of residence } \\
\hline $1.1-2.5$ vs. 1 year & $1.52(1.04-2.22)$ & 0.029 & $1.39(0.88-2.19)$ & 0.156 \\
\hline $2.6-4.5$ vs. 1 year & $2.25(1.53-3.31)$ & $<0.001$ & $1.44(0.90-2.29)$ & 0.127 \\
\hline 4.6- vs. 1 year & $3.18(2.19-4.63)$ & $<0.001$ & $1.91(1.22-3.02)$ & 0.005 \\
\hline \multicolumn{5}{|l|}{ Degree of care dependency } \\
\hline $4-6$ vs. $<4$ & $12.97(5.02-33.51)$ & $<0.001$ & $9.93(3.63-27.13)$ & $<0.001$ \\
\hline $7-9$ vs. $<4$ & $81.49(32.02-207.40)$ & $<0.001$ & $50.06(18.54-135.15)$ & $<0.001$ \\
\hline $10-12$ vs. $<4$ & $530.82(183.67-1,534.09)$ & $<0.001$ & 294.25 (94.37-917.45) & $<0.001$ \\
\hline Mobility limitations, yes vs. no & $7.55(5.31-10.74)$ & $<0.001$ & $3.46(2.35-5.10)$ & $<0.001$ \\
\hline Verbal agitation, yes vs. no & $1.48(1.11-1.98)$ & 0.007 & $0.87(0.58-1.29)$ & 0.474 \\
\hline Physical agitation, yes vs. no & $1.61(1.11-2.32)$ & 0.012 & $1.13(0.68-1.86)$ & 0.642 \\
\hline \multicolumn{5}{|l|}{ Fall } \\
\hline Within the last 30 days, yes vs. no & $0.98(0.68-1.40)$ & 0.902 & & \\
\hline Between $>30$ and 180 days, yes vs. no & $1.23(0.91-1.65)$ & 0.175 & & \\
\hline
\end{tabular}

Residents excluded from model due to missing values: Mobility limitations $(n=38)$, physical agitation $(n=1)$, verbal agitation $(n=2)$. ${ }^{\text {FTE }}$ full time equivalent

mentioned rather rarely and are a surprising result, since recent studies indicated these characteristics as positively associated with restraint use [3]. However, this remains speculative, since comparisons with commonly used tools like the Neuropsychiatric Inventory (NPI) are lacking.

Only one organisational characteristic (residents per caregiver) turned out to be positively associated with physical restraint use in the univariate model. However, this association did not remain significant in the multiple model. Therefore, none of the organisational characteristics were significantly associated with physical restraint use. These results are in accordance with several earlier studies $[2,6,34]$. However, other studies indicate that physical restraints are less likely in dementia-specific care units $[12,35,36]$ and in nursing homes with non-profit ownership [9,37]. In general, the literature is inconsistent 
concerning associations of organisational factors with physical restraint use. Nevertheless, residents' characteristics seem to have a greater influence on physical restraints use than organisational characteristics.

The persistent use of physical restraints in nursing homes requires effective interventions for educating nursing staff as well as all persons involved, e.g. residents, relatives, nursing experts and nursing homes directors. As recent studies have demonstrated, multicomponent interventions may reduce physical restraints in nursing homes $[17,19]$. Since the results of our study are comparable to former studies from Germany $[2,6]$, the transfer and implementation of an effective intervention programme developed for nursing homes in Germany [17] might be appropriate.

With regard to the implementation of evidence-based practice, we have to take into consideration the role of nursing leadership. Effective leadership is vital in this process as well as the institution and the culture in which the leader operates [38]. Current evidence shows the relationship between positive relational leadership and lower physical restraint use [39]. Leaders can facilitate working conditions, create an atmosphere of open communication with staff and promote positive relationships among nurses in order to ensure safe, high-quality patient care and work engagement [40]. These components seem to be equally important to successfully reduce physical restraints.

\section{Strengths and limitations}

One of the strengths of the current study is the inclusion of a large sample comprising 1362 residents from two culturally different regions of one country. The results provide the basis for better understanding of the practice of physical restraint use in Switzerland. However, we cannot exclude that the results are not exactly transferable to the other parts of the country. Our study contributes to the body of knowledge by adding new evidence from a sound association analysis of residentrelated and organization-related characteristics. Personal visits to the nursing homes in order to explain in detail the performance of the study and the data collection ensured a trustworthy relationship with the nursing staff, which is a very important premise for research in an ethically sensitive area. The number of missing values was low $(n=41)$, which demonstrates an accurately performed data collection.

We did not collect the data by direct observation which is certainly the most valid method. However, as shown in validation studies, medical or residents' records are a reliable source of information [41].

Former studies have shown that cognitive impairment is associated with the use of physical restraints [3]. Unfortunately, we could not explore a potential association since the data on cognitive status were not eligible for regression analysis.

\section{Conclusion}

Our study results show substantial variation in the frequency of physical restraint use between nursing homes. This suggests that a reduction could be possible and the use of bedrails could be minimized in order to respect the persons' autonomy and mobility. The new Swiss law may lead to more awareness with regard to physical restraints but further efforts towards restraint-free nursing care are required. Therefore, it seems to be worthwhile to explore the approach of nursing homes with particularly low and high use of physical restraints in future qualitative research. The development of interventions should consider specific residents' characteristics associated with physical restraints.

Generally, the use of physical restraints must always consider the serious consequences for residents, their dignity and how their quality of life might be affected. The decision-making process concerning the application of physical restraints has always to respect the resident's situation as well as nurse- and organisation-related factors. Leadership could essentially contribute to restraint-free nursing care by sensitizing nurses and creating optimal working conditions.

\begin{abstract}
Abbreviations
ADL: Activities of daily living; AOR: Adjusted odds ratio; BESA: Bedarfsklärungs- und Abrechnungs-System (in English: Needs Assessment and Payoff System); Cl: Confidence interval; DF: Design factor; FTE: Full time equivalent; ICCC: Intracluster correlation coefficient; MAQ: Maastricht attitude questionnaire; n: Number of characteristic values; NPI: Neuropsychiatric inventory; RAl: Resident assessment instrument; SAS: Statistical analysis systems; SD: Standard deviation; SPSS: Statistic package for social sciences; vs: Versus.
\end{abstract}

Competing interests

The authors declare that they have no competing interests.

\section{Authors' contributions}

$\mathrm{HH}, \mathrm{ES}$ and GM designed the study. $\mathrm{HH}$ and ES led and performed the data collection, GM acted as supervisor. $\mathrm{HH}$ and ES analysed and interpreted the data and prepared the manuscript drafts. $\mathrm{BH}$ performed the statistical analyses. GM and $\mathrm{BH}$ commented on all drafts. All authors read and approved the final manuscript.

\section{Acknowledgements}

The authors sincerely thank the directors and all study coordinators of the twenty nursing homes for their participation in this research; Manuela Eicher, PhD, University of Applied Sciences and Arts Western Switzerland, School of Health, Fribourg and Diana Staudacher, PhD, University of Applied Sciences, St.Gallen for advice counselling; Dorothea Weber and Julian Hirt, study assistants, University of Applied Sciences, St.Gallen, for assistance in data collection.

This study was supported by grants from the Käthe-Zingg-Schwichtenberg-Fonds, Switzerland, the Nursing Science Foundation Switzerland, Basel, the University of Applied Sciences, St.Gallen, Department of Health, St.Gallen, Switzerland, as well as the University of Applied Sciences and Arts Western Switzerland, School of Health, Fribourg, Switzerland, which are gratefully acknowledged. 


\section{Author details}

${ }^{1}$ Department of Health, University of Applied Sciences, Rosenbergstrasse 59, P.O. BoxCH-9001 St.Gallen, Switzerland. ²Department of Nursing Science, Witten/Herdecke University, Witten, Germany. ${ }^{3}$ School of Health, University of Applied Sciences and Arts Western Switzerland, Fribourg, Switzerland. ${ }^{4}$ mediStatistica, Neuenrade, Germany. ${ }^{5}$ Institute of Health and Nursing Science, Medical Faculty, Martin Luther University Halle-Wittenberg, Halle (Saale), Germany.

\section{Received: 26 March 2015 Accepted: 12 October 2015}

\section{Published online: 21 October 2015}

\section{References}

1. Castle NG, Engberg J. The health consequences of using physical restraints in nursing homes. Med Care. 2009;47(11):1164-73. doi:10.1097/MLR.0b013e3181b58a69.

2. Heinze C, Dassen T, Grittner U. Use of physical restraints in nursing homes and hospitals and related factors: A cross-sectional study. J Clin Nurs. 2012;21(7-8):1033-40. doi:10.1111/j.1365-2702.2011.03931.x.

3. Hofmann H, Hahn S. Characteristics of nursing home residents and physical restraint: a systematic literature review. J Clin Nurs. 2013;23:3012-24. doi:10.1111/jocn.12384.

4. Feng Z, Hirdes JP, Smith TF, Finne-Soveri H, Chi I, Du Pasquier J-N, et al. Use of physical restraints and antipsychotic medications in nursing homes: A cross-national study. Int J Geriatr Psychiatry. 2009;24(10):1110-8.

5. Zúñiga F, Ausserhofer D, Serdaly C, Bassal C, De Geest S, Schwendimann R. SHURP. Schlussbericht zur Befragung des Pflege- und Betreuungspersonals in Alters- und Pflegeinstitutionen der Schweiz. Basel: Institut für Pflegewissenschaft, Universität Basel; 2013.

6. Meyer G, Köpke S, Haastert B, Mühlhauser I. Restraint use among nursing home residents: Cross-sectional study and prospective cohort study. J Clin Nurs. 2009;18(7):981-90. doi:10.1111/j.1365-2702.2008.02460.x.

7. Retsas AP. Survey findings describing the use of physical restraints in nursing homes in Victoria, Australia. Int J Nurs Stud. 1998;35(3):184-91.

8. Gastmans C, Milisen K. Use of physical restraint in nursing homes: clinical-ethical considerations. J Med Ethics. 2006;32(3):148-52. doi:10.1136/jme.2005.012708.

9. Engberg J, Castle NG, McCaffrey D. Physical restraint initiation in nursing homes and subsequent resident health. Gerontologist. 2008:48(4):442-52.

10. Möhler R, Meyer G. Attitudes of nurses towards the use of physical restraints in geriatric care: A systematic review of qualitative and quantitative studies. Int J Nurs Stud. 2014;51(2):274-88. doi:10.1016/j.jinurstu.2013.10.004.

11. Huizing A, Hamers J, de Jonge J, Candel M, Berger M. Organisational determinants of the use of physical restraints: A multilevel approach. Soc Sci Med. 2007;65(5):924-33.

12. Kirkevold O, Engedal K. Quality of care in Norwegian nursing homesdeficiencies and their correlates. Scand J Caring Sci. 2008;22(4):560-7.

13. Baier R, Butterfield K, Patry G, Harris Y, Gravenstein S. Identifying star performers: The relationship between ambitious targets and nursing home quality improvement. J Am Geriatr Soc. 2009;57(8):1498-503.

14. Scherder EJA, Bogen T, Eggermont LHP, Hamers JPH, Swaab DF. The more physical inactivity, the more agitation in dementia. Int Psychogeriatr. 2010;22(8):1203-8.

15. Healey F, Oliver D, Milne A, Connelly JB. The effect of bedrails on falls and injury: a systematic review of clinical studies. Age Ageing. 2008;37(4):368-78. doi:10.1093/ageing/afn112.

16. Möhler R, Richter T, Köpke S, Meyer G. Interventions for preventing and reducing the use of physical restraints in long-term geriatric care - a Cochrane review. J Clin Nurs. 2012;21(21-22):3070-81.

17. Köpke S, Mühlhauser I, Gerlach A, Haut A, Haastert B, Möhler R, et al. Effect of a guideline-based multicomponent intervention on use of physical restraints in nursing homes: a randomized controlled trial. JAMA. 2012;307(20):2177-84. doi:10.1001/jama.2012.4517.

18. Gulpers MJM, Bleijlevens MHC, Ambergen T, Capezuti E, van Rossum E, Hamers JPH. Belt restraint reduction in nursing homes: Effects of a multicomponent intervention program. J Am Geriatr Soc. 2011;59(11):2029-36.

19. Gulpers MJM, Bleijlevens MHC, Ambergen T, Capezuti E, van Rossum E, Hamers JPH. Reduction of Belt Restraint Use: Long-Term Effects of the EXBELT Intervention. J Am Geriatr Soc. 2013;61(1):107-12. doi:10.1111/jgs.12057.
20. Gulpers MJM, Bleijlevens MHC, Capezuti E, van Rossum E, Ambergen T, Hamers JPH. Preventing belt restraint use in newly admitted residents in nursing homes: A quasi-experimental study. Int J Nurs Stud. 2012;49(12):1473-9.

21. Schweizerische Eidgenossenschaft. Schweizerisches Zivilgesetzbuch ZGB. 2008.

22. De Vries OJ, Ligthart GJ, Nikolaus T. Differences in period prevalence ot the use of physical restraints in elderly inpatients of European hospitals and nursing homes. J Gerontol Ser A. 2004;59(9):M922-3. doi:10.1093/gerona/59.9.M922.

23. Donner A, Klar N. Design and Analysis of Cluster Randomization Trials in Health Research. Hoboken: Wiley; 2000.

24. Hamers JPH, Meyer G, Koepke S, Lindemann R, Groven R, Huizing AR. Attitudes of Dutch, German and Swiss nursing staff towards physical restraint use in nursing home residents, a cross-sectional study. Int J Nurs Stud. 2009;46(2):248-55. doi:10.1016/j.jinurstu.2008.06.007.

25. Haut A, Kolbe N, Strupeit S, Mayer H, Meyer G. Attitudes of relatives of nursing home residents toward physical restraints. J Nurs Scholar. 2010;42(4):448-56. doi:10.1111/j.1547-5069.2010.01341.x

26. BESA-Care. System BESA. Leistungskatalog LK2010. http://www.besacare.ch/ index.cfm/07E0CEA4-0EFE-7325-D55E9DECE0A4233F/. (n.d.). Accessed 11 March 2013

27. Q-Sys AG. RAI-NH Pflegedokumentation. http://www.qsys.ch. (n.d.) Accessed 11 March 2013

28. Schweizerische Eidgenossenschaft. Bundesgesetz über die Neuordnung der Pflegefinanzierung. 2008.

29. Kerry SM, Bland JM. Unequal cluster sizes for trials in English and Welsh general practice: implications for sample size calculations. Stat Med. 2001;20(3):377-90.

30. Brown H, Prescott R. Applied Mixed Models in Medicine. Statistics in Practice. 2nd ed. Hoboken: John Wiley \& Sons; 2006.

31. Beerens HC, Sutcliffe C, Renom-Guiteras A, Soto ME, Suhonen R, Zabalegui A, et al. Quality of life and quality of care for people with dementia receiving long term institutional care or professional home care: the european righttimeplacecare study. J Am Med Dir Assoc. 2014;15(1):54-61. doi:10.1016/j.jamda.2013.09.010.

32. National Patient Safety Agency. Bedrails - Reviewing the Evidence: A Systematic Literature Review. London: NPSA; 2007.

33. Bredthauer D, Becker C, Eichner B, Koczy P, Nikolaus T. Factors relating to the use of physical restraints in psychogeriatric care: a paradigm for elder abuse. Z Gerontol Geriatr. 2005;38(1):10-8. doi:10.1007/s00391-005-0285-y.

34. Chiba Y, Yamamoto-Mitani N, Kawasaki M. A national survey of the use of physical restraint in long-term care hospitals in Japan. J Clin Nurs. 2012;21(9-10):1314-26.

35. Lai C, Yeung J, Mok V, Chi I. Special care units for dementia individuals with behavioural problems. Cochrane Database Syst Rev 2009(4). doi:10.1002/14651858.CD006470.pub2

36. Nobili A, Piana I, Balossi L, Pasina L, Matucci M, Tarantola M, et al. Alzheimer special care units compared with traditional nursing home for dementia care are there differences at admission and in clinical outcomes? Alzheimer Dis Assoc Disord. 2008;22(4):352-61.

37. Castle NG, Engberg J. Staff turnover and quality of care in nursing homes. Med Care. 2005:43(6):616-26.

38. Sandstrom B, Borglin G, Nilsson R, Willman A. Promoting the implementation of evidence-based practice: a literature review focusing on the role of nursing leadership. Worldviews Evid Based Nurs. 2011;8(4):212-23. doi:10.1111/j.1741-6787.2011.00216.x.

39. Wong CA, Cummings GG, Ducharme L. The relationship between nursing leadership and patient outcomes: a systematic review update. J Nurs Manag. 2013;21(5):709-24. doi:10.1111/jonm.12116.

40. Cummings GG, MacGregor T, Davey M, Lee H, Wong CA, Lo E, et al. Leadership styles and outcome patterns for the nursing workforce and work environment: A systematic review. Int J Nurs Stud. 2010;47(3):363-85. doi:10.1016/j.jphurstu.2009.08.006.

41. Laurin D, Voyer $P$, Verreault $R$, Durand P. Physical restraint use among nursing home residents: A comparison of two data collection methods. BMC Nurs. 2004;3(1):1-7. doi:10.1186/1472-6955-3-5. 